\title{
Research Article pth Mean Practical Stability for Large-Scale Itô Stochastic Systems with Markovian Switching
}

\author{
Yan Yun, ${ }^{1}$ Huisheng Shu, ${ }^{1}$ and Yan $C_{h}{ }^{2,3}$ \\ ${ }^{1}$ Department of Applied Mathematics, Donghua University, Shanghai 201620, China \\ ${ }^{2}$ Department of Electronics and Information Engineering, Putian University, Fujian Putian 351100, China \\ ${ }^{3}$ College of Information Sciences and Technology, Donghua University, Shanghai 201620, China
}

Correspondence should be addressed to Huisheng Shu, huisheng.shu@gmail.com

Received 29 June 2011; Accepted 6 September 2011

Academic Editor: Zidong Wang

Copyright (C) 2012 Yan Yun et al. This is an open access article distributed under the Creative Commons Attribution License, which permits unrestricted use, distribution, and reproduction in any medium, provided the original work is properly cited.

\begin{abstract}
Motivated by the study of a class of large-scale stochastic systems with Markovian switching, this correspondence paper is concerned with the practical stability in the $p$ th mean. By investigating Lyapunov-like functions and the basic comparison principle, some criteria are derived for various types of practical stability in the $p$ th mean of nonlinear stochastic systems. The main contribution of these results is to convert the problem of practical stability in the $p$ th mean of stochastic systems into the one of practical stability of the comparative deterministic systems.
\end{abstract}

\section{Introduction}

The practical dynamical systems in real-world applications, such as engineering, physics, and economics, usually exhibit the stochastic nature due to the uncertain resulting from the external environment. Examples include traffic systems, flexible manufacturing systems, and economic systems. All of these dynamical systems can be modeled by Itô stochastic differential equations. Therefore, it is not surprising that various problems of Itô stochastic differential equations have received considerable attention. As the development of the stochastic theory including stochastic processes, stochastic integral, and stochastic partial differential equations, the theory on the stochastic differential equations in infinite dimensional spaces has advanced greatly, and, so far, a rich body of literature has been reported.

Stability is one of the most important issues in the analysis and synthesis of stochastic systems and often regarded as the first characteristic of the dynamical systems (or models) to be studied. Currently, there have already been many kinds of stability concepts such as asymptotic stability, stability in probability, almost sure exponential stability, and mean-square 
exponential stability. These stabilities are analyzed in terms of the theory of functional analysis which, however, makes it difficult for a newcomer to enter this interesting and important field. The concepts of stability mentioned above are defined in the sense of Lyapunov. In many real-world applications, however, the systems might not be asymptotically stable in the sense of Lyapunov and stay nearby a state with an acceptable fluctuation. For example, an aircraft or a missile may oscillate around a mathematically unstable course, and its performance may be acceptable yet. To treat with such situations, a new stability concept, that is, the practical stability, has been proposed by LaSalle and Lefschetz [1] and, subsequently, has been developed in [2-4].

With respect to stochastic systems, the practical stability in the $p$ th mean and the stability in probability have been introduced in [5] and [6], respectively. In these papers, both the stochastic system and its corresponding auxiliary equation have deterministic initial conditions. By using the powerful comparison theorem, which is developed in $[7,8]$, these concepts have been extended to a more general class of stochastic systems in [9], where the resulting auxiliary system has random initial conditions. Recently, stochastic differential equations with Markovian switching have also stirred a great deal of research interests due primarily to their insight into applications. The fundamental theory of existence and uniqueness of the solution of stochastic differential equations with Markovian switching has been studied well in $[10,11]$, while the stability issues have been investigated in [12-15].

To be more specific, during the research of practical stability, one of the foremost challenges to system theory in the present-day advanced technological world is to overcome the increasing size and complexity of the corresponding mathematical models $[9,16]$. This is the so-called large-scale systems, which is more closed to the actual circumstances, and, therefore, it has a wider significance. However, since the computational efforts are enormous, the practical stability of large-scale systems has received relatively little attention. Recently, several important results have been obtained in the area of practical stability for large-scale Itô stochastic systems, see [17], for example, in which the large amount of computational efforts of a large-scale complex system become simpler and more economical by decomposition into a number of interconnected subsystems; these subsystems, to some extent, can be considered to be independent so that some of the qualitative behaviors of the corresponding subsystems can be combined with interconnection constraints to come up with the qualitative behavior of the overall large-scale systems. Like all other systems, the stability issue should be examined first in the large-scale systems. However, the problem of $p$ th mean practical stability for large-scale Itô stochastic systems with Markovian switching has not been addressed properly, which motives the current research.

In this paper, the problem of the $p$ th mean practical stability for large-scale Itô stochastic systems with Markovian switching is studied. First, the notion of practical stability in the $p$ th mean is introduced and extended for the large-scale stochastic systems with Markovian switching. Then, the concepts of Lyapunov-like vector-valued functions coupled with the decomposition-aggregation techniques are utilized to develop a comparison principle. In addition, some general criteria of practical stability for the large-scale Itô stochastic systems with Markovian switching are obtained. Finally, an example is given to show the usefulness of the developed criteria.

\section{Preliminaries}

Let $\left\{\Omega, \mathcal{F}, \mathcal{F}_{t \geq 0}, P\right\}$ be a complete probability space with a filtration satisfying the usual conditions, that is, the filtration is continuous on the right and $\mathcal{F}_{0}$ contains all $P$-zero sets. 
$W(t)=\left(W_{1}(t), W_{2}(t), \ldots, W_{m}(t)\right)^{T}$ is an $m$-dimensional Wiener process defined on the probability space. $\left\{r(t), t \in R_{+}\right\}$denotes a right-continuous Markov chain on the probability space $\left\{\Omega, \mathcal{F}, \mathscr{F}_{t \geq 0}, P\right\}$, taking values in a finite state space $S=\{1,2, \ldots, N\}$ with generator $\Gamma=$ $\left(\gamma_{i j}\right)_{N \times N}$ given by

$$
P\{r(t+\Delta)=j \mid r(t)=i\}= \begin{cases}\gamma_{i j} \Delta+o(\Delta), & \text { if } i \neq j, \\ 1+\gamma_{i i} \Delta+o(\Delta), & \text { if } i=j,\end{cases}
$$

where $\Delta>0$ and $\gamma_{i j} \geq 0$ is the transition rate from $i$ to $j$ if $i \neq j$ while $\gamma_{i i}=-\Sigma_{j \neq i} \gamma_{i j}$. We assume that the Markov chain $r(\cdot)$ is independent of Wiener process $W(\cdot)$. It is known that almost every sample path of $r(t)$ is a right-continuous step function with a finite number of simple jumps in any finite subinterval of $R$.

Consider the following Itô stochastic differential equations with Markovian switching:

$$
d x(t)=f(t, r(t), x(t)) d t+\sigma(t, r(t), x(t)) d w(t)
$$

with the initial condition $x_{0}=\xi$. Here $\xi$ is assumed to be independent of $W(\cdot)$. The nonlinear functions $f: \mathcal{R}_{+} \times S \times \mathcal{R}^{n} \rightarrow \mathcal{R}^{n}$ and $\sigma: \mathcal{R}_{+} \times S \times \mathcal{R}^{n} \rightarrow \mathcal{R}^{n \times m}$ satisfy Lipschitz condition and Linear growth condition.

In this paper, we always assume that (2.2) has a unique continuous solution $x(t, \xi)$ such that $E\left(\sup \|x(t, \xi)\|^{p}\right)<\infty$ for each $t \geq 0$ and $p \geq 0$. Further, it is also assumed that $f(t, j, 0)=0$ and $\sigma(t, j, 0)=0$ for all $j \in S$ and, accordingly, (2.2) has a trivial solution $x(t, 0) \equiv 0$.

Now, we decompose (2.2) into $n$ interconnected subsystems described by

$$
\begin{gathered}
d x_{i}=\left[p_{i}\left(t, r(t), x_{i}\right)+h_{i}(t, r(t), x)\right] d t+\sum_{r=1}^{m}\left[l_{r}^{i}\left(t, r(t), x_{i}\right)+k_{r}^{i}(t, r(t), x)\right] d w_{r}(t), \\
x_{i}\left(t_{0}\right)=x_{0}, \quad i=1,2, \ldots, n,
\end{gathered}
$$

where nonlinear functions $p_{i}\left(t, r(t), x_{i}\right)$, and $l_{r}^{i}\left(t, r(t), x_{i}\right) \in \mathcal{R}_{+} \times S \times \mathcal{R} \rightarrow \mathcal{R}, h_{i}(t, r(t), x)$ and $k_{r}^{i}(t, r(t), x) \in \mathcal{R}_{+} \times S \times \mathcal{R}^{n} \rightarrow \mathcal{R}$, respectively.

\section{Comparison Principle}

The comparison principle has proved to be a useful tool in the study of the qualitative and quantitative properties of solution processes of Itô-type stochastic system with Markovian switching. In this section, by employing the concept of Lyapunov vector-valued function coupled with decomposition-aggregation techniques, and together with the theory of differential inequalities, the comparison theorems for the large-scale system (2.3) are developed.

The $\mathcal{L}$-operator for the $n$-interconnected subprocedures (2.3) is defined as

$$
\begin{aligned}
\mathcal{L}^{c} V_{i}(t, j, x(t))= & \frac{\partial V_{i}(x, t, j)}{\partial t}+\sum_{j=1}^{n}\left(\frac{\partial V_{i}(x(t), t, j)}{\partial x_{j}}\right)\left[p_{j}\left(t, j, x_{i}\right)+h_{j}(t, j, x)\right] \\
& +\frac{1}{2} \sum_{j, r=1}^{n} \frac{\partial V_{i}^{2}(t, j, x)}{\partial x_{j} \partial x_{r}} a_{j r}+\sum_{j=i}^{N} \gamma_{i j} V_{i}(t, j, x),
\end{aligned}
$$


where

$$
\begin{array}{r}
\left(a_{j r}\right)=l_{j}^{i}\left(t, i, x_{i}\right)^{T} l_{r}^{i}\left(t, i, x_{i}\right)+l_{j}^{i}\left(t, i, x_{i}\right)^{T} k_{r}^{i}(t, i, x)+k_{j}^{i}(t, i, x)^{T} l_{r}^{i}\left(t, i, x_{i}\right)+k_{j}^{i}(t, i, x)^{T} k_{r}^{i}(t, i, x), \\
j, r=1,2, \ldots, n, \quad s=1,2, \ldots, N .
\end{array}
$$

Now, consider now the following auxiliary random differential system:

$$
d u=g(t, u) d t, \quad u\left(t_{0}\right)=u_{0},
$$

where $g(t, u) \in \mathcal{C}\left[\partial \times \mathcal{R}_{+}^{n}, \mathbb{R}^{n}\right]$ is concave and quasi-monotone nondecreasing in $u$ for each fixed $t \in 2$ and $u_{0}$ is an $n$-dimensional random vector. Let $u\left(t, t_{0}, u_{0}\right)$ be any solution of the system (3.3) and $r\left(t, t_{0}, u_{0}\right)$ the maximal solution process of the system (3.3) through $\left(t_{0}, u_{0}\right)$.

We need the following corresponding definitions of practical stability for the auxiliary system (3.3).

Definition 3.1. System (3.3) is said to be practically stable, if for all given $(\lambda, \mathcal{A})$ with $0<\lambda<$ $\mathcal{A}, E \sum_{i=1}^{n} u_{i_{0}}<\mathcal{\lambda}$ implies $\sum_{i=1}^{n} u_{i}\left(t, t_{0}, u_{0}\right)<\mathcal{A}$, for all $t \geq t_{0}$ for some $t_{0} \in \mathcal{R}_{+}$.

Definition 3.2. Set $\alpha=\left(\alpha_{1}, \alpha_{2}, \ldots, \alpha_{s}\right)$ and $\beta=\left(\beta_{1}, \beta_{2}, \ldots, \beta_{s}\right)$. If $\alpha_{i} \leq \beta_{i}, \forall i=1,2, \ldots, s$, one denotes $\alpha<\beta$.

In this section, by employing the Lyapunov-like functions and the basic comparison principle of stochastic systems, some results on various types of practical stability in the $p$ th mean are obtained for the interconnected system (2.3).

Theorem 3.3. Assume that there exist functions $V_{i}(t, x)$ and $g(t, u)$ satisfying the following conditions:

(i) for every $V_{i}(t, x) \in \mathcal{C}\left[\partial \times \mathcal{R}^{n}, \mathcal{R}_{+}\right], \partial V_{i}(t, x) / \partial t, \partial V_{i}(t, x) / \partial x^{2}$, and $\partial^{2} V_{i}(t, x) / \partial x^{2}$ exist and are continuous for $(t, x) \in\left(2 \times R^{n}\right)$, and $\mathcal{L} V_{i}(t, j, x) \leq g_{i}\left(t, V_{i}(t, j, x)\right)$ holds for all $(t, x) \in 2 \times \mathcal{R}^{n}, i=1,2, \ldots, n, j=1,2, \ldots, N$;

(ii) $g(t, u) \in \mathcal{C}\left[2 \times \mathcal{R}^{n}, \boldsymbol{R}_{+}^{n}\right]$ is a quasi-monotone nondecreasing concave function in $u$ for each $t \in 2$, and satisfies $g(t, 0) \equiv 0$;

(iii) the maximal solution of the auxiliary differential system (3.3), that is, $r\left(t, t_{0}, u_{0}\right)$ exists for all $t \geq t_{0}$, where $u_{0}$ is an $n$-dimensional random vector;

(iv) for the solution process $x_{i}(t)=x_{i}\left(t, t_{0}, x_{0}\right)$ of system (2.3), if $E\left[V_{i}(t, x(t), j)\right]$ exists for all $t \geq t_{0}$, then

$$
E[V(t, x(t), j)] \leq r\left(t, t_{0}, u_{0}\right)
$$

whenever $t \geq t_{0}$ and $V\left(t_{0}, x_{0}, j\right) \leq u_{0}$, where

$$
V(t, x, j)=\left[V_{1}(t, x, j), V_{2}(t, x, j), \ldots, V_{n}(t, x, j)\right]^{T}
$$


Proof. By applying generalized Itô formula, we have



Setting $m_{i}(t)=E V_{i}(x, t, j)$, we obtain

$$
m_{i}(t)-m_{i}\left(t_{0}\right)=\int_{t_{0}}^{t} E \bumpeq V_{i}(x, s, j) d_{s}
$$

based on which, letting $\rho \rightarrow 0^{+}$, it can be obtained that

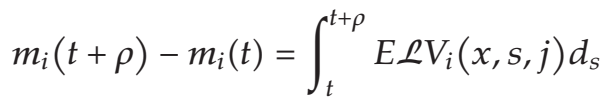

$$
\begin{aligned}
& \leq \int_{t}^{t+\rho} E g_{i}\left(t, V_{i}(x, s, j)\right) d_{s} \\
& \leq \int_{t}^{t+\rho} g_{i}\left(t, E V_{i}(x, s, j)\right) d_{s} .
\end{aligned}
$$

That is, for each $i=1,2, \ldots, n$, we have

$$
D^{+} m_{i}(t) \leq g_{i}\left(t, m_{i}(t)\right)
$$

where $D^{+}$denotes the upper right Dini-derivative operator and $m_{i}(t)=E V_{i}(x, t, j)$.

Denoting $D^{+} m(t)=\left(D^{+} m_{1}(t), D^{+} m_{2}(t), \ldots, D^{+} m_{n}(t)\right)$, we have

$$
D^{+} m(t) \leq g(t, m(t))
$$

According to comparison theorem in [17], it immediately follows that

$$
E[V(t, x(t), j)] \leq r\left(t, t_{0}, u_{0}\right)
$$

whenever $t \geq t_{0}$ and $V\left(t_{0}, x_{0}, j\right) \leq u_{0}$, and hence the proof is complete.

\section{Practical Stability Criteria}

In this section, by employing Lyapunov-like functions and basic comparison principles for interconnected systems developed in the previous section, we give various types of practical stability in the $p$ th mean of the interconnected system (2.3).

Definition 4.1. System (2.3) is said to be practically stable in the $p$ th mean, if for all given $(\lambda, \mathcal{A})$ with $0<\lambda<\mathcal{A}, E\left\|x_{0}\right\|^{p}<\lambda$ implies $E\left\|x\left(t, t_{0}, x_{0}\right)\right\|^{p}<\mathcal{A}$ for all $t \geq t_{0}$. 
Definition 4.2. A function $\varphi(u)$ is said to belong to the class $\mathcal{K}$, if $\varphi \in \mathcal{K}\left(\mathcal{R}_{+}, \mathcal{R}_{+}\right)$satisfies $\varphi(0)=0 ; \varphi(t, u)$ is said to belong to the class $\mathcal{C} \mathcal{K}$, if $\varphi \in \mathcal{C} \mathcal{K}\left(\mathcal{R}_{+} \times \mathcal{R}_{+}, \mathcal{R}_{+}\right)$is continuous, concave, and strictly increasing in $u$ for each $t \in \mathcal{R}_{+}$and satisfies $\varphi(t, 0)=0 ; \varphi(u)$ is said to belong to the class $\mathcal{V K}$, if $\varphi \in \mathcal{V K}\left[\mathcal{R}_{+}, \mathcal{R}_{+}\right]$is continuous, convex, and strictly increasing for each $u \in \mathcal{R}_{+}$and satisfies $\varphi(0)=0$.

The following theorem gives the criteria of practical stability.

Theorem 4.3. Assume that

(i) all the hypotheses of Theorem 3.3 hold;

(ii) for $(t, x) \in 2 \times \mathcal{R}^{n}$, the following inequality holds:

$$
b\left(\|x\|^{p}\right) \leq \sum_{i=1}^{n} V_{i}(t, x, j) \leq a\left(t,\|x\|^{p}\right),
$$

where $b \in \mathcal{U} \mathcal{K}\left[\mathcal{R}_{+}, \boldsymbol{R}_{+}\right]$and $a \in \mathcal{C} \mathcal{\mathcal { K }}\left[\mathcal{R}_{+}, \boldsymbol{R}_{+}\right]$satisfy $b(0)=0$ and $a(t, 0)=0$, respectively;

(iii) the maximal solutions of the auxiliary differential system (3.3) on $t \geq t_{0}$, that is, $r(t)=$ $r\left(t, t_{0}, u_{0}\right)$ and $E\left[r\left(t, t_{0}, u_{0}\right)\right]$ both exist;

(iv) for the given $\lambda$ and $\mathcal{A}(0<\lambda<\mathcal{A})$, system (3.3) is practically stable with $a\left(t_{0}, \lambda\right)<b(\mathcal{A})$.

Then, the interconnected system (2.3) is practically stable in the $p$ th mean.

Proof. Let $x_{i}(t)=x_{i}\left(t, t_{0}, x_{0}\right)(i=1,2, \ldots, n)$ be any solution process of system (2.3). It can be obtained from Theorem 3.3 that

$$
E[V(t, x(t), j)] \leq r\left(t, t_{0}, u_{0}\right)
$$

whenever $t \geq t_{0}$ and $V\left(t_{0}, x_{0}, j\right) \leq u_{0}$, where $r\left(t, t_{0}, u_{0}\right)$ is the maximal solution of system (3.3) on $\left[t_{0}, \infty\right)$, and thus,

$$
\sum_{i=1}^{n} E\left[V_{i}(t, j, x(t))\right] \leq \sum_{i=1}^{n} r_{i}\left(t, t_{0}, u_{0}\right)
$$

Considering that $r\left(t, t_{0}, u_{0}\right)$ is practically stable, we have

$$
\sum_{i=1}^{n} u_{i_{0}}<a\left(t_{0}, \lambda\right)
$$

which implies that

$$
\sum_{i=1}^{n} r_{i}\left(t, t_{0}, u_{0}\right)<b(\mathcal{A}) \quad \forall t \geq t_{0} .
$$


We claim that $E\left\|x_{0}\right\|^{p}<\lambda$ implies $E\left\|x\left(t, t_{0}, x_{0}\right)\right\|^{p}<\mathcal{A}$, for all $t \geq t_{0}$ for some $t_{0} \in \mathcal{R}_{+}$, where $x(t)=\left(x_{1}(t), x_{2}(t), \ldots, x_{n}(t)\right)$ is any solution of system (2.3) with $E\left\|x_{0}\right\|^{p}<\lambda$. Suppose that this claim is not true, then there exists a $t_{1}>t_{0}$ and a solution $x(t)=x\left(t, t_{0}, x_{0}\right)$ of system (2.3) with $E\left\|x_{0}\right\|^{p}<\lambda$ satisfying

$$
E\left\|x\left(t_{1}\right)\right\|^{p}=\mathcal{A}, \quad E\|x(t)\|^{p}<A \quad \text { for } t_{0} \leq t<t_{1} .
$$

Then, it follows from assumption (ii) that

$$
\sum_{i=1}^{n} E\left[V_{i}\left(t, j, x\left(t_{1}\right)\right)\right] \geq b\left(E\left\|x\left(t_{1}\right)\right\|^{p}\right)=b(\mathcal{A})
$$

Choosing $u_{0}$ such that $V\left(t_{0}, x_{0}\right)=u_{0}$ and $\sum_{i=1}^{n} u_{i_{0}}=a\left(t_{0}, E\left\|x_{0}\right\|^{p}\right)$, we have

$$
\sum_{i=1}^{n} E\left[V_{i}\left(t_{1}, j, x\left(t_{1}\right)\right)\right] \leq \sum_{i=1}^{n} r_{i}\left(t_{1}, t_{0}, u_{0}\right)<b(\mathcal{A})
$$

Therefore, we arrive at the following contradiction:

$$
b(\mathcal{A}) \leq \sum_{i=1}^{n} E\left[V_{i}\left(t_{1}, j, x\left(t_{1}\right)\right)\right]<b(\mathcal{A}) .
$$

This completes the proof.

Theorem 4.4. Assume the following:

(i) all hypotheses of Theorem 3.3 hold;

(ii) for $(t, x) \in \tau \times \mathbb{R}^{n}, V(t, x)$ satisfies the following inequality:

$$
\Phi\left(\|x\|^{p}\right) \leq \sum_{i=1}^{n} V_{i}(t, x, j)
$$

where $\Phi \in \mathcal{U} \mathcal{K}\left[\boldsymbol{R}_{+}, \boldsymbol{R}_{+}\right]$and $\boldsymbol{\mho K}\left[\boldsymbol{R}_{+}, \boldsymbol{R}_{+}\right]$is the collection of all continuous, convex, and increasing functions defined on $\boldsymbol{R}_{+}$into itself with $\Phi(0)=0$;

(iii) the maximal solution $r(t)$ of system (3.3) through $\left(t_{0}, u_{0}\right)$ converges to the zero vector as $t \rightarrow \infty$. Then, the interconnected system (2.3) is practically stable in the pth mean.

Proof. An application of Theorem 3.3 gives the following inequality:

$$
E V(t, j, x(t)) \leq r\left(t, t_{0}, u_{0}\right)
$$

where $r\left(t, t_{0}, u_{0}\right)$ is the maximal solution of system (3.3).

From assumption (ii), it can be seen that $\Phi(\lambda)>0$ for every $A>\lambda>0$. Let $\lambda$ be a positive real number satisfying $\left\|x_{0}\right\|^{p}<\lambda$. By choosing $u_{0}$ such that $V\left(t_{0}, x_{0}\right)=u_{0}$ and noting as- 
sumption (ii) and the continuity of $V$, there exists $\lambda^{*}$ such that $\left\|x_{0}\right\|^{p}<\lambda$ implies $\sum_{i=1}^{n} E\left[V_{i}\left(t, j, x_{0}\right)\right] \leq \lambda^{*}$.

From assumption (iii) and the practical stability of $r(t)$ of system (3.3), we have $\sum_{i=1}^{n} V_{i}\left(t, j, x_{0}\right)=\sum_{i=1}^{n} u_{i_{0}}<\lambda^{*}$ which implies that

$$
\sum_{i=1}^{n} r_{i}\left(t, t_{0}, u_{0}\right)<\Phi(\mathcal{A})
$$

It follows from (4.11) that

$$
\sum_{i=1}^{n} E\left[V_{i}(t, j, x(t))\right] \leq \sum_{i=1}^{n} r_{i}\left(t, t_{0}, u_{0}\right), \quad t \geq t_{0},
$$

and from (4.10), we arrive at the following inequality:

$$
E\left[\Phi\left(\|x\|^{p}\right)\right] \leq \sum_{i=1}^{n} E\left[V_{i}(t, j, x(t))\right]
$$

By noting that $\Phi$ is a convex function and using the Tensen's inequality [18], we have

$$
\Phi\left[E\left(\|x\|^{p}\right)\right] \leq E\left[\Phi\left(\|x\|^{p}\right)\right] .
$$

Then, from (4.12)-(4.15), we obtain

$$
\Phi\left[E\left(\|x\|^{p}\right)\right]<\Phi(\mathcal{A})
$$

whenever $\left\|x_{0}\right\|^{p}<\lambda$, which implies

$$
E\left[\left(\|x(t)\|^{p}\right)\right]<\mathcal{A}, \quad \forall t \geq t_{0} .
$$

Thus the proof is complete.

\section{Example}

Consider the following stochastic differential equation with Markovian switching:

$$
d x(t)=f(t, r(t), x(t)) d t+\sigma(t, r(t), x(t)) d w(t),
$$

where $x=\left[\begin{array}{ll}x_{1} & x_{2}\end{array}\right]^{T} \in \mathcal{R}^{2}$ is the sate vector, $w(t)$ is a normalized scalar wiener process.

The nonlinear vector function $\sigma=\left[\begin{array}{ll}\sigma_{1} & \sigma_{2}\end{array}\right]^{T} \in \mathcal{C}\left[\mathcal{R}_{+} \times \mathcal{S} \times \mathcal{R}^{2}, \mathbb{R}^{2}\right]$ satisfies $\sigma(t, r(t), 0) \equiv 0$,

$$
\begin{aligned}
& {\left[\sigma_{1}(t, r(t), x(t))+\sigma_{2}(t, r(t), x(t))\right]^{2} \leq\left(x_{1}+x_{2}\right)^{2} \lambda(t),} \\
& {\left[\sigma_{1}(t, r(t), x(t))-\sigma_{2}(t, r(t), x(t))\right]^{2} \leq\left(x_{1}-x_{2}\right)^{2} \lambda(t),}
\end{aligned}
$$

where $\lambda \in \mathcal{C}\left[\mathcal{R}_{+}, \mathcal{R}_{+}\right] \cap L_{1}[0, \infty)$. 
The nonlinear function $f(t, r(t), x(t))$ is taken as

$$
f(t, r(t), x(t))=\left(\begin{array}{c}
e^{-t}+\sin (t) x_{1} \\
e^{-t}+\sin (t) x_{2}
\end{array}\right)
$$

We choose

$$
V(t, r(t), x)=\left(\begin{array}{l}
V_{1}(t, x) \\
V_{2}(t, x)
\end{array}\right)=\left(\begin{array}{c}
\left(x_{1}+x_{2}\right)^{2} \\
\left(x_{1}-x_{2}\right)^{2}
\end{array}\right)
$$

as the Lyapunov function for (5.1). It is not difficult to obtain

$$
|x|^{2} \leq \sum_{i=1}^{2} V_{i}(t, r(t), x(t)) \leq 2|x|^{2}
$$

Now, consider the following auxiliary random differential system:

$$
d u=g(t, u) d t \quad u\left(t_{0}\right)=u_{0}
$$

where $u \in R^{2}, g(t, u)$ is given by

$$
g(t, u)=\left(\begin{array}{l}
\left(2 e^{-t}+2 \sin (t)+\lambda(t)\right) u_{1} \\
\left(2 e^{-t}+2 \sin (t)+\lambda(t)\right) u_{2}
\end{array}\right)
$$

We obtain

$$
\mathcal{L} V_{i}(t, r(t), x(t)) \leq g_{i}\left(t, V_{i}(t, r(t), x(t))\right) \quad \text { for }(t, x) \in \mathcal{R}_{+} \times \mathcal{R}^{2}
$$

let $a(r)=b(r)=r$. Obviously, the functions $a(r)$ and $b(r)$ are both convex and concave. Moreover, it is easy to see that $g(t, u)$ is concave and quasi-monotone nondecreasing in $u$ for the fixed $t$, and hence the system (5.1) is uniformly practically stable.

\section{Conclusions}

In this paper, the notion of practical stability in the $p$ th mean is introduced and extended for the large-scale stochastic systems with Markovian switching. By employing Lyapunov-like functions and the basic comparison principle, sufficient conditions are established for various types of practical stability in the $p$ th mean of nonlinear stochastic systems. The advantage of these results is to convert the problem of practical stability in the $p$ th mean of stochastic systems into the problem of practical stability of the comparative deterministic systems. Future research topics include the investigation on the filtering and control problems for uncertain nonlinear stochastic systems, see for example, [19-28]. 


\section{Acknowledgment}

This work is supported by the National Natural Science Foundation of China (No. 60974030) and the Science and Technology Project of Education Department in Fujian Province, China (No. JA11211).

\section{References}

[1] J. P. LaSalle and S. Lefschetz, Stability Theory by Liapunovs Direct Method with Applications, Academic Press, New York, NY, USA, 1961.

[2] A. A. Martynyuk, Practical Stability of Motion, Naukav a Dumka, Kiev, Ukraine, 1983.

[3] A. A. Martynyuk, "Averaging method in the theory of motion stability," Nonlinear Vibration Problems, vol. 14, pp. 71-79, 1973.

[4] V. Laksmikantham, S. Leela, and A. A. Martynyuk, Practical Stability of Nonlinear Systems, World Scientific, Singapore, 1990.

[5] Z. S. Feng, Y. Q. Liu, and F. W. Guo, "Criteria for practical stability in the $p$-th mean of nonlinear stochastic systems," Applied Mathematics and Computation, vol. 49, no. 2-3, pp. 251-260, 1992.

[6] A. H. Tsoi and B. Zhang, "Practical stabilities of Itô type nonlinear stochastic differential systems and related control problems," Dynamic Systems and Applications, vol. 6, no. 1, pp. 107-124, 1997.

[7] G. S. Ladde and B. A. Lawrence, "Stability and convergence of large scale stochastic approximation procedures," International Journal of Systems Science, vol. 26, no. 3, pp. 595-618, 1995.

[8] L. Shaikhet, "Stability of stochastic differential systems with Markovian Switching," Stochastic Processes, vol. 2, no. 18, pp. 180-184, 1996.

[9] S. Sathananthan, Practical Stability Criteria for Nonlinear Ito-Type Stochastic Control Systems, Academic Press, New York, NY, USA, 2000.

[10] M. A. O. Xuerong, A. Matasov, and A. B. Piunovskiy, "Stochastic differential delay equations with Markovian switching," Bernoulli, vol. 6, no. 1, pp. 73-90, 2000.

[11] P. Zhao, "Practical stability, controllability and optimal control of stochastic Markovian jump systems with time-delays," Automatica, vol. 44, no. 12, pp. 3120-3125, 2008.

[12] X. Mao, "Robustness of stability of stochastic differential delay equations with Markovian Switching," Stability and Control: Theory and Applications, vol. 3, no. 1, pp. 48-61, 2000.

[13] H. Gilsing, "On the stability of the Euler scheme for an affine stochastic delay differential equation with the delay," Tech. Rep. 20,SFB 373, Humboldt University, Berlin, Germany, 2001.

[14] A. V. Swishchuk and Y. I. Kazmerchuk, "Stability of stochastic of differential delay Ito's equations with Possion Jumps and with Markovian Switching. Application to Financial Models," Teoriya Veroyatnostei i Matematicheskaya Statistika, vol. 63 (63), 2001.

[15] J. Luo, J. Zou, and Z. Hou, "Comparison principle and stability criteria for stochastic differential delay equations with Markovian switching," Science in China. Series A, vol. 46, no. 1, pp. 129-138, 2003.

[16] D. D. Siljak, Large-Scale Dynamic Systems: Stability and Structure, vol. 3 of North-Holland Series in System Science and Engineering, North-Holland, New York, NY, USA, 1979.

[17] S. Sathananthan and S. Suthaharan, "Practical stability criteria for large-scale nonlinear stochastic systems by decomposition and aggregation," Dynamics of Continuous, Discrete \& Impulsive Systems. Series A, vol. 8, no. 2, pp. 227-248, 2001.

[18] G. S. Ladde and V. Lakshmikantham, Random Differential Inequalities, vol. 150 of Mathematics in Science and Engineering, Academic Press, New York, NY, USA, 1980.

[19] H. Dong, Z. Wang, D. W.C. Ho, and H. Gao, "Robust $H_{\infty}$ filtering for Markovian jump systems with randomly occurring nonlinearities and sensor saturation: the finite-horizon case," IEEE Transactions on Signal Processing, vol. 59, no. 7, pp. 3048-3057, 2011.

[20] Y. Tang, Z. Wang, W. K. Wong, J. Kurths, and J. Fang, "Multiobjective synchronization of coupled systems," Chaos, vol. 21, no. 2, Article ID 025114, 2011.

[21] Z. Wang, J. Lam, L. Ma, Y. Bo, and Z. Guo, "Variance-constrained dissipative observer-based control for a class of nonlinear stochastic systems with degraded measurements," Journal of Mathematical Analysis and Applications, vol. 377, no. 2, pp. 645-658, 2011.

[22] B. Shen, Z. Wang, Y. S. Hung, and G. Chesi, “Distributed $H_{\infty}$ filtering for polynomial nonlinear stochastic systems in sensor networks," IEEE Transactions on Industrial Electronics, vol. 58, no. 5, pp. 1971-1979, 2011. 
[23] J. Liang, Z. Wang, and X. Liu, "Distributed state estimation for discrete-time sensor networks with randomly varying nonlinearities and missing measurements," IEEE Transactions on Neural Networks, vol. 22, no. 3, pp. 486-496, 2011.

[24] X. He, Z. Wang, Y. D. Ji, and D. H. Zhou, "Robust fault detection for networked systems with distributed sensors," IEEE Transactions on Aerospace and Electronic Systems, vol. 47, no. 1, pp. 166-177, 2011.

[25] B. Shen, Z. Wang, and X. Liu, "Bounded $H_{\infty}$ synchronization and state estimation for discrete timevarying stochastic complex networks over a finite horizon," IEEE Transactions on Neural Networks, vol. 22, no. 1, pp. 145-157, 2011.

[26] B. Shen, Z. Wang, H. Shu, and G. Wei, " $H_{\infty}$ filtering for uncertain time-varying systems with multiple randomly occurred nonlinearities and successive packet dropouts," International Journal of Robust and Nonlinear Control, vol. 21, no. 14, pp. 1693-1709, 2011.

[27] Z. Wang, D. W. C. Ho, H. Dong, and H. Gao, "Robust $H_{\infty}$ finite-horizon control for a class of stochastic nonlinear time-varying systems subject to sensor and actuator saturations," IEEE Transactions on Automatic Control, vol. 55, no. 7, pp. 1716-1722, 2010.

[28] Z. Wang, Y. Liu, and X. Liu, “Exponential stabilization of a class of stochastic system with Markovian jump parameters and mode-dependent mixed time-delays," IEEE Transactions on Automatic Control, vol. 55, no. 7, pp. 1656-1662, 2010. 


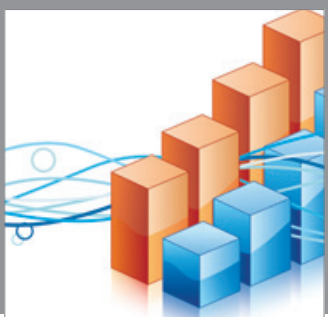

Advances in

Operations Research

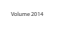

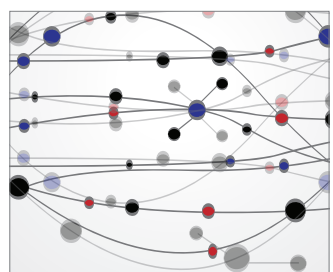

\section{The Scientific} World Journal
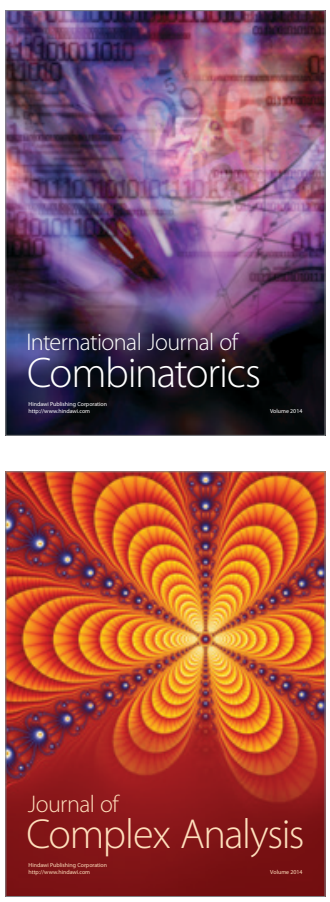

International Journal of

Mathematics and

Mathematical

Sciences
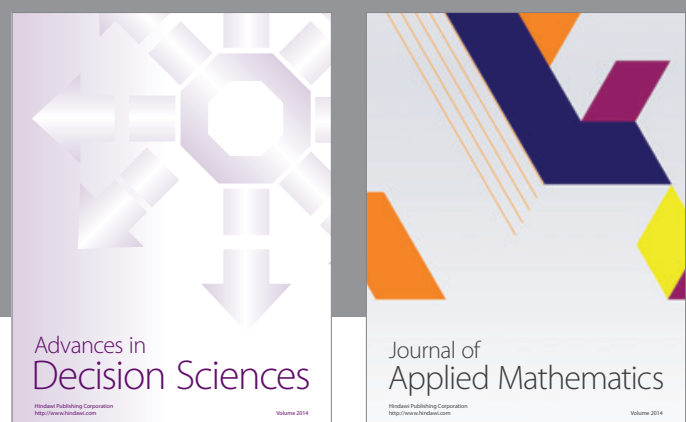

Journal of

Applied Mathematics
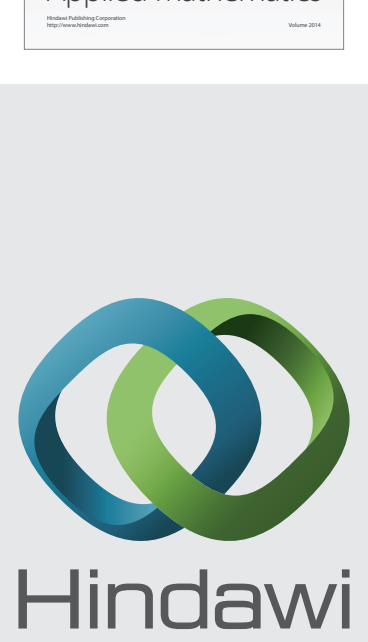

Submit your manuscripts at http://www.hindawi.com
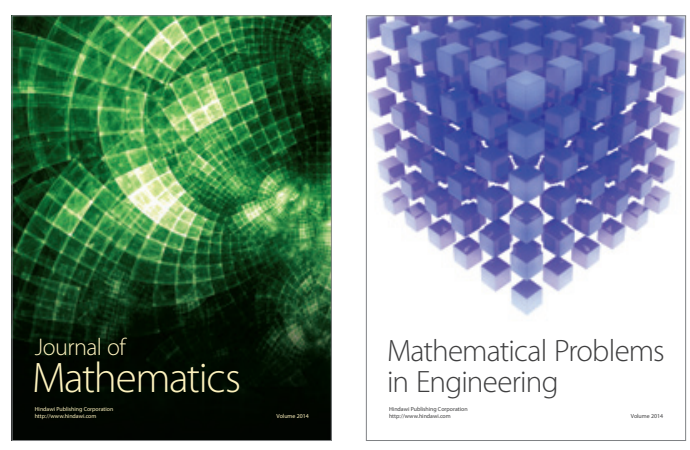

Mathematical Problems in Engineering


Journal of

Function Spaces
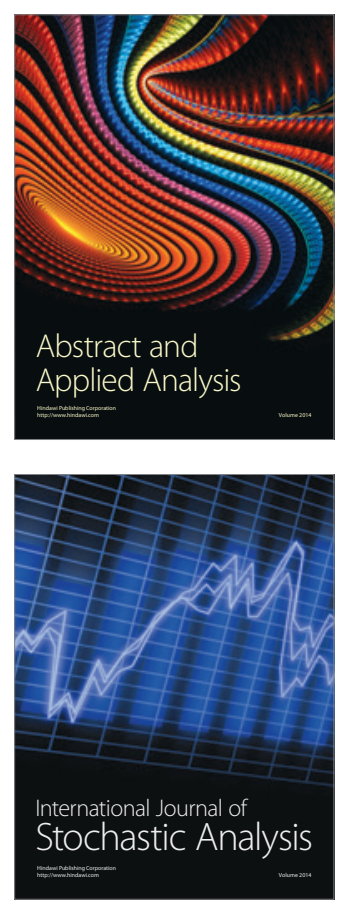

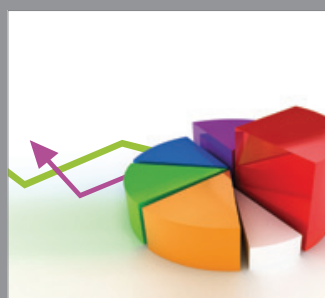

ournal of

Probability and Statistics

Promensencen
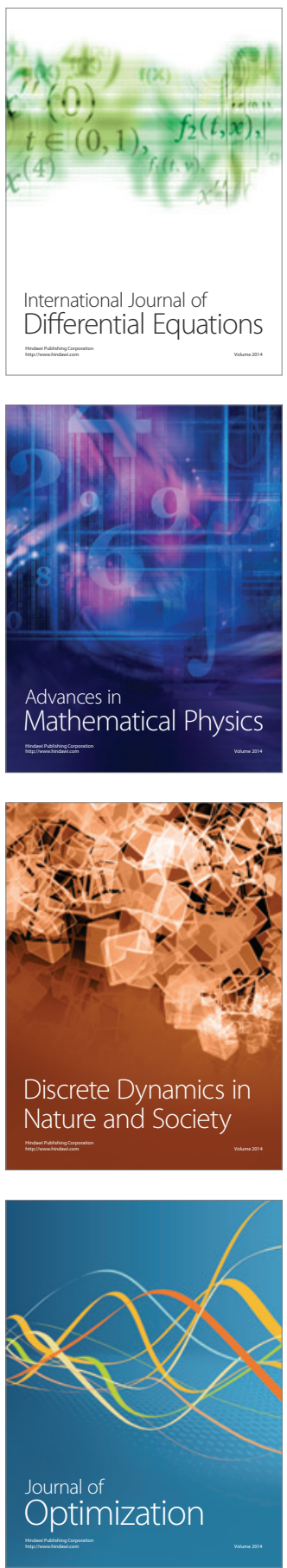